Supporting Information

\title{
On the Mechanism of Human Saliva Interaction With Environmental Dust in Relation to Spreading of Viruses
}

\author{
Bekir Sami Yilbas ${ }^{1,2,3^{*}} \quad$ Ghassan Hassan $^{1,4} \quad$ Ayse Elif Yilbas $^{5} \quad$ Abba A. Abubakar ${ }^{1}$ \\ Hussain Al-Qahtani ${ }^{1}$ \\ ${ }^{1}$ Mechanical Engineering Department, KFUPM, Dhahran 31261, Saudi Arabia \\ ${ }^{2}$ Center of Research Excellence in Renewable Energy (CoRE-RE), KFUPM, Dhahran 31261, \\ Saudi Arabia \\ ${ }^{3}$ Senior Researcher at K.A.CARE Energy Research \& Innovation Center, DTV \\ Dhahran 31261, Saudi Arabia \\ ${ }^{4}$ Researcher at K.A.CARE Energy Research \& Innovation Center, DTV, \\ Dhahran 31261, Saudi Arabia \\ 55University of Ottawa, General surgery, Ottawa, Canada \\ *Correspondence and requests for materials should be addressed to B.S.Y. (email: \\ bsyilbas@kfupm.edu.sa)
}

The supplementation information contains:

Number of pages $=11$

Number of figures $=5$

Number of tables $=1$

Table of contents

Page

S1. Mathematical Formulation for the Numerical Model S2-S8

S2. C-T Scan Image of Dust Particle S9

S3. Variation of saliva residue with dust size factor S10

S4. References 


\section{S1. Mathematical Formulation for the Numerical Model}

The impact of saliva droplet on a stationary dust particle is modeled by adopting the Navier-Stokes' equation for laminar flow. The liquid (saliva) droplet is considered to be incompressible Newtonian fluid, while the surrounding air is considered to be weaklycompressible due to its low Mach number. In line with experimental study, the dust particle is considered to be a rigid (stationary) solid body. Thus, the impact simulation is carried out using the continuity and momentum equations expressed in Eq. S1 and S2.

The continuity equation can be expressed as:

$\frac{\partial \rho}{\partial t}+\nabla \cdot(\rho \bar{v})=0$ Eq. S1

The momentum equation can be expressed as:

$\rho \frac{\partial \bar{v}}{\partial t}+\rho(\bar{v} \cdot \nabla) \bar{v}=\nabla \cdot\left[-p \bar{I}+\mu\left(\nabla \bar{v}+(\nabla \bar{v})^{T}\right)-\left(\frac{2 \mu}{3}-\kappa\right)(\nabla \cdot \bar{v}) \bar{I}\right]+\rho \bar{g}+\gamma m \delta \bar{n}+\bar{F}_{r} E q . S 2$

here: $\rho$ is density, $\bar{v}$ is velocity field vector, $\bar{g}$ is gravitational acceleration, $p$ is pressure, $\gamma$ is liquidgas surface tension coefficient, $\mu$ is the fluids dynamic viscosity, $m=\frac{\bar{n}}{|\bar{n}|}$ is curvature, $\bar{n}$ is the unit normal vector at the saliva-air interface, $\delta$ is the Dirac-Delta function, $\bar{F}_{r}$ is friction source term, and $t$ is time.

To model the movement of the saliva droplet within the stagnant air phase, the conserved level-set (two-phase) flow model is utilized as expressed in Eq. S3.

$\frac{\partial \varphi}{\partial t}+\bar{v} \cdot \nabla \varphi=\zeta \cdot \nabla \cdot\left(\varepsilon \nabla \varphi-\varphi(1-\varphi) \frac{\nabla \varphi}{|\nabla \varphi|}\right)$ Eq. S3

here: $\varphi$ is level-set function, $\bar{v}$ is velocity vector, $\zeta$ is re-initialization parameter, $\varepsilon$ is the parameter that controls interface thickness, and $t$ is time. The level-set function $(\varphi)$ denotes the saliva phase 
fraction within the domain. Hence, it is used to scale the fluid properties (such as density and viscosity) according to mixtures rules.

\section{Initial conditions}

The initial size and location of the saliva droplet and dust particles are defined according to the experimental data. In order to capture accurately the impacting droplet behaviour, the rules of similitude were used to estimate the droplet size and initial velocity mimicking the interaction between the actual human saliva droplet and dust particles. Utilizing the Froud number relation, the initial velocity of the saliva droplet can be obtained from the relation, $\frac{V_{\text {rel }}}{V_{\text {exp }}}=\sqrt{\sqrt{\frac{l_{x p}}{l_{\text {rel }}}}}$, where $V$ is the droplet initial velocity, $l$ is the droplet size (diameter). Incorporating the actual droplet velocity $(3 \mathrm{~m} / \mathrm{s})$ and size $(50 \mu \mathrm{m})$ emanating from the human mouth, the experimental droplet velocity for $800 \mu \mathrm{m}$ droplet becomes $0.75 \mathrm{~m} / \mathrm{s}$. Hence, the saliva droplet of size $1.2 \mu \mathrm{L}$ is utilized in the simulations (Fig. S1) and the droplet is considered to be under gravitational influence and released with an initial velocity of $0.75 \mathrm{~m} / \mathrm{s}$.

Hence, the initial conditions used for the current analysis are expressed as:

$$
\begin{aligned}
& \bar{v}(x, y, 0)=\left\{\begin{array}{c}
0.75 \mathrm{~m} / \mathrm{s} \text { for saliva droplet } \\
0 \mathrm{~m} / \mathrm{s} \text { for air }
\end{array}\right. \\
& p(x, y, 0)=\left\{\begin{array}{l}
\frac{2 \gamma}{R} \text { for saliva droplet } \\
0 \text { for air }
\end{array}\right. \\
& \varphi(x, y, 0)=\left\{\begin{array}{rrr}
1 & \text { for saliva droplet } \\
& 0.5 & \text { for interface }
\end{array}\right. \\
&
\end{aligned}
$$




\section{$\underline{\text { Boundary conditions }}$}

Boundaries 1, 2, 3 and 4 are defined to be pressure outlet boundaries (Fig. S1). Boundary 5 resembles the interface between the saliva droplet and air. It is considered to be freely deform within the domain. Boundary 6 (Fig. S1) is the wetted wall in which the dynamic contact angle between saliva droplet, air, and the dust particle surface are defined as adopted in previous works $^{1,2}$. Furthermore, adhesion/frictional forces which cause sticking of saliva are defined at boundary 6 . Hence, the adopted boundary conditions can be expressed as:

$p(x, y, t)=0$ (outlet condition on boundaries $1,2,3$ and 4$)$ Eq. S7

$\bar{v}(x, y, t) \cdot \bar{n}_{w}=0 \quad$ (slipping wall on boundary 6$)$ Eq. S8

Here, $\bar{n}_{w}$ is unit normal vector at the wetted wall.

$\bar{F}_{f r}(x, y, t)=\gamma\left(\bar{n}_{w}-\left(\bar{n} \delta \cos \theta-\frac{\mu}{h} \bar{v}(x, y, t)\right)\right)($ friction force on boundary 6) Eq. S9

Here, $\bar{n}$ is unit normal vector at the water-air interface, $\theta$ is the dynamic contact angle, $h$ is slipping length.

$\left(-p I+\mu\left(\nabla \bar{v}+(\nabla \bar{v})^{T}\right)\right) \bar{n}=0 \quad($ Slip condition on boundary (5)) Eq. S10

Here: $I$ is the identity matrix, the superscript $T$ is the matrix transpose, $\bar{v}$ is velocity vector, and $\bar{n}$ is normal unit vector.

$\bar{v} \cdot \bar{n}=0 ; \bar{K}-(\bar{K} \cdot \bar{n})=0 \quad$ (No fluid penetration on boundary (5)) Eq. S11

Where, $\bar{K}=\mu\left(\nabla \bar{v}+(\nabla \bar{v})^{T}\right) \bar{n}$ 


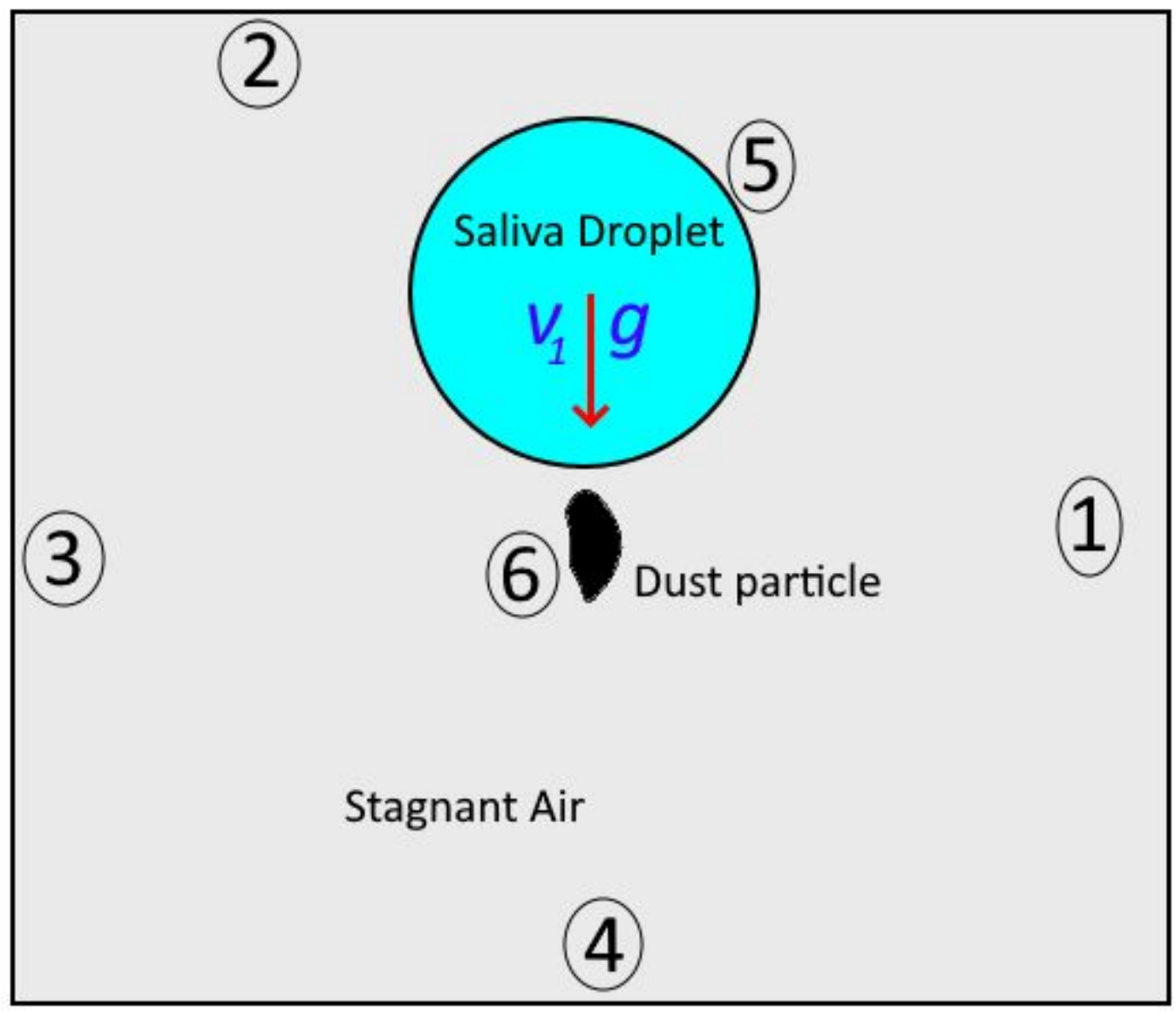

Figure S1. Boundaries conditions adopted for the simulations: Pressure outlet is defined at boundaries 1, 2, 3 and 4. Boundary 5 resembles the interface between the saliva droplet and ambient air, which is free to deform. Boundary 6 is wetted wall where contact angle and adhesive (frictional) forces are defined.

Table S1. Material properties adopted in the simulations.

\begin{tabular}{lcl}
\hline Parameter & Saliva & Air \\
\hline Density, $\rho\left(\mathrm{kg} / \mathrm{m}^{3}\right)$ & 1012 & 1.2 \\
Viscosity, $\mu(\mathrm{Pa} \mathrm{s})$ & $1.29 \times 10^{-3}$ & $1.813 \times 10^{-5}$ \\
Surface tension, $\sigma(\mathrm{N} / \mathrm{m})$ & 0.057 & - \\
Dynamic contact angle & $\theta_{\mathrm{adv}}=38.6^{0}, \theta_{\mathrm{rec}}=36.5^{0}$
\end{tabular}




\section{$\underline{\text { Numerical implementation }}$}

COMSOL multi-physics code ${ }^{3}$ is adopted for the numerical implementation of the model with the initial and boundary conditions presented in Eq. S4 - S11. Since the simulation of the impact of saliva on real dust particle in 3-dimensions requires large computational efforts, a 2dimensional model is adopted for the current study. Furthermore, for effective numerical convergence, the second-order Euler backward difference scheme is used to discretize the time derivatives in the flow. Also, time steps as small as $10^{-11} \mathrm{~s}$ are adopted for the numerical solution. Similarly, a regular mesh of sufficient density containing about 83,465 triangular elements (with an average quality of 0.98 ) is adopted for the simulations (Fig. S2). For effective numerical convergence, the level-set reinitialization parameter $(\zeta)$ is adopted be equal to the maximum velocity of flow $(1 \mathrm{~m} / \mathrm{s})$; while the interface thickness $(\varepsilon)$ is taken as half of the maximum element (edge) length. As demonstrated in Fig.S1, the numerical solution obtained for the $1.2 \mu \mathrm{L}$ saliva droplet impacting on the real dust particle is independent of the mesh size adopted (Fig. S3). 


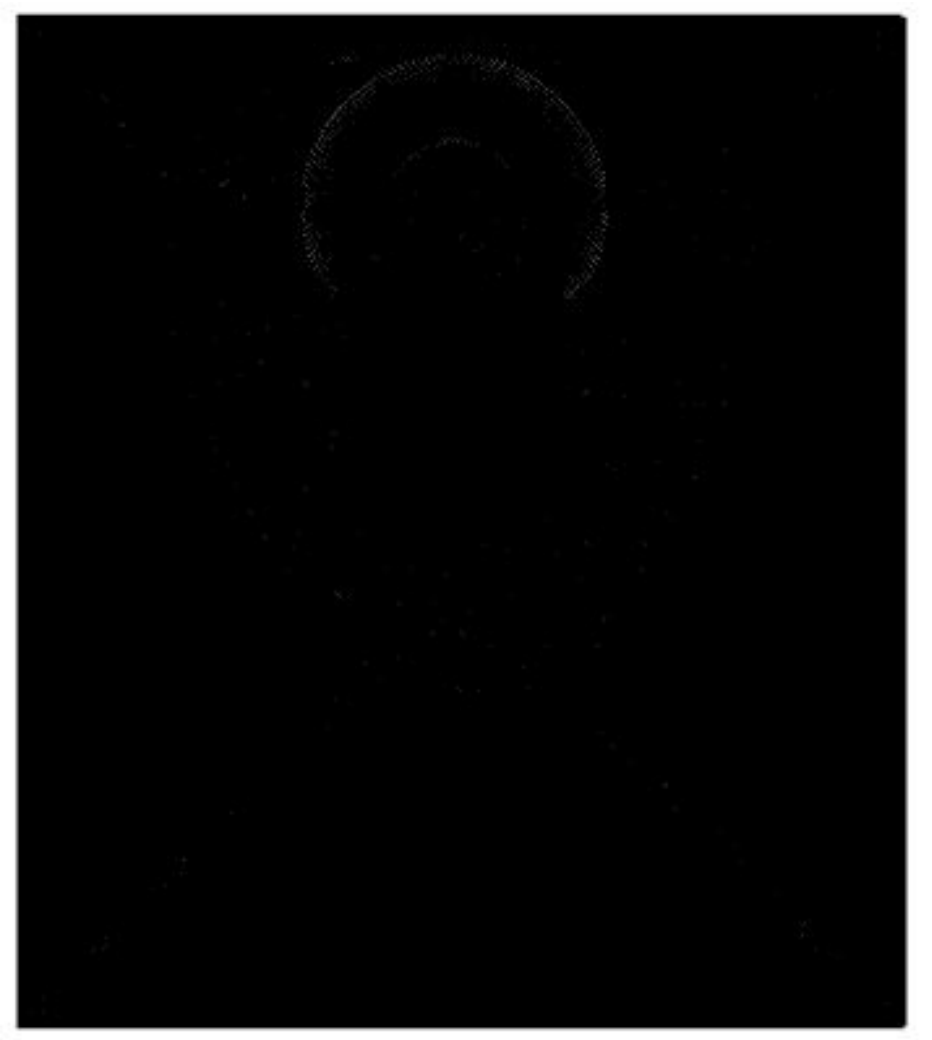

a)
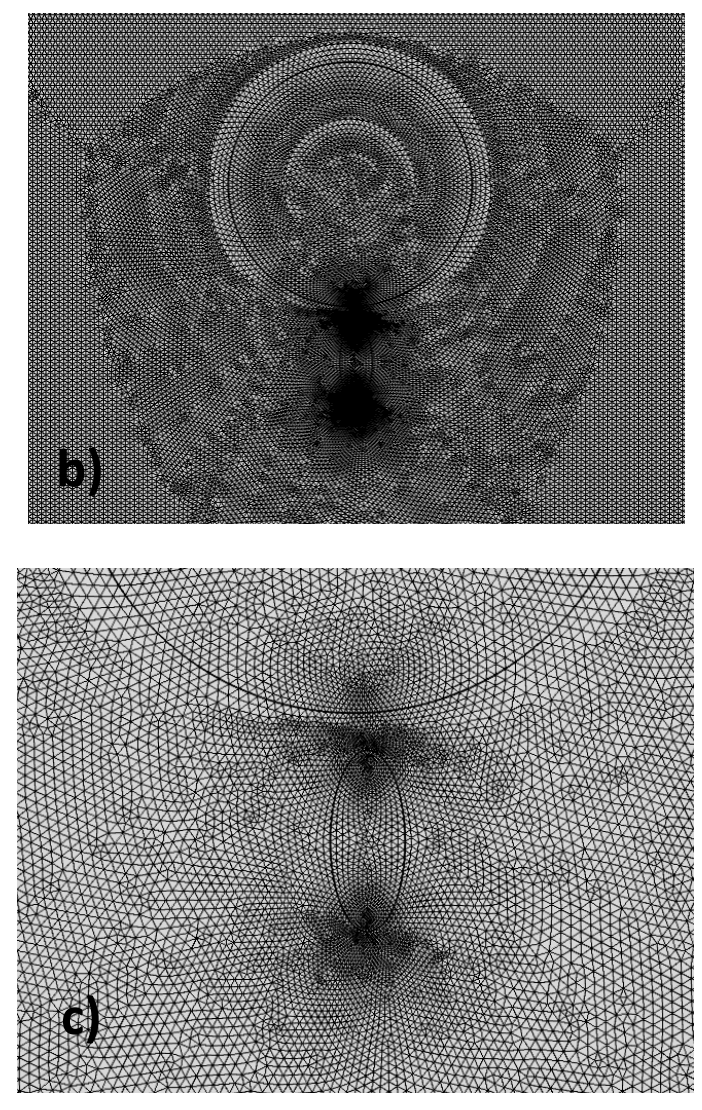

Figure S2. (a) FE mesh utilized for the study, (b) initial droplet and dust particle locations, (c) mesh type near an ellipsoidal dust particle 

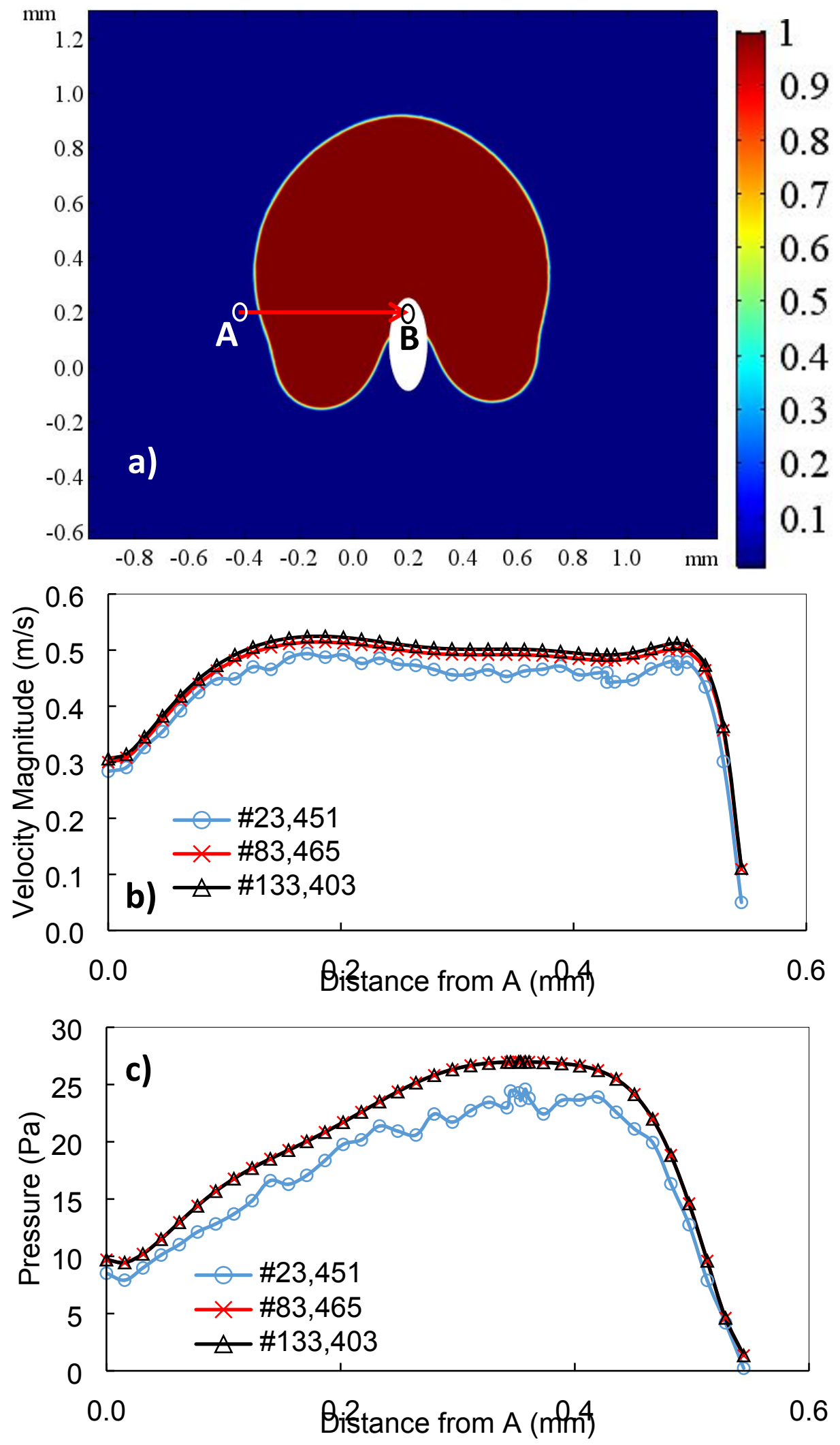

Figure S3. (a) Line cut along which mesh independent test is conducted, (b) Variation of velocity with mesh size, (c) Variation of pressure with mesh size 


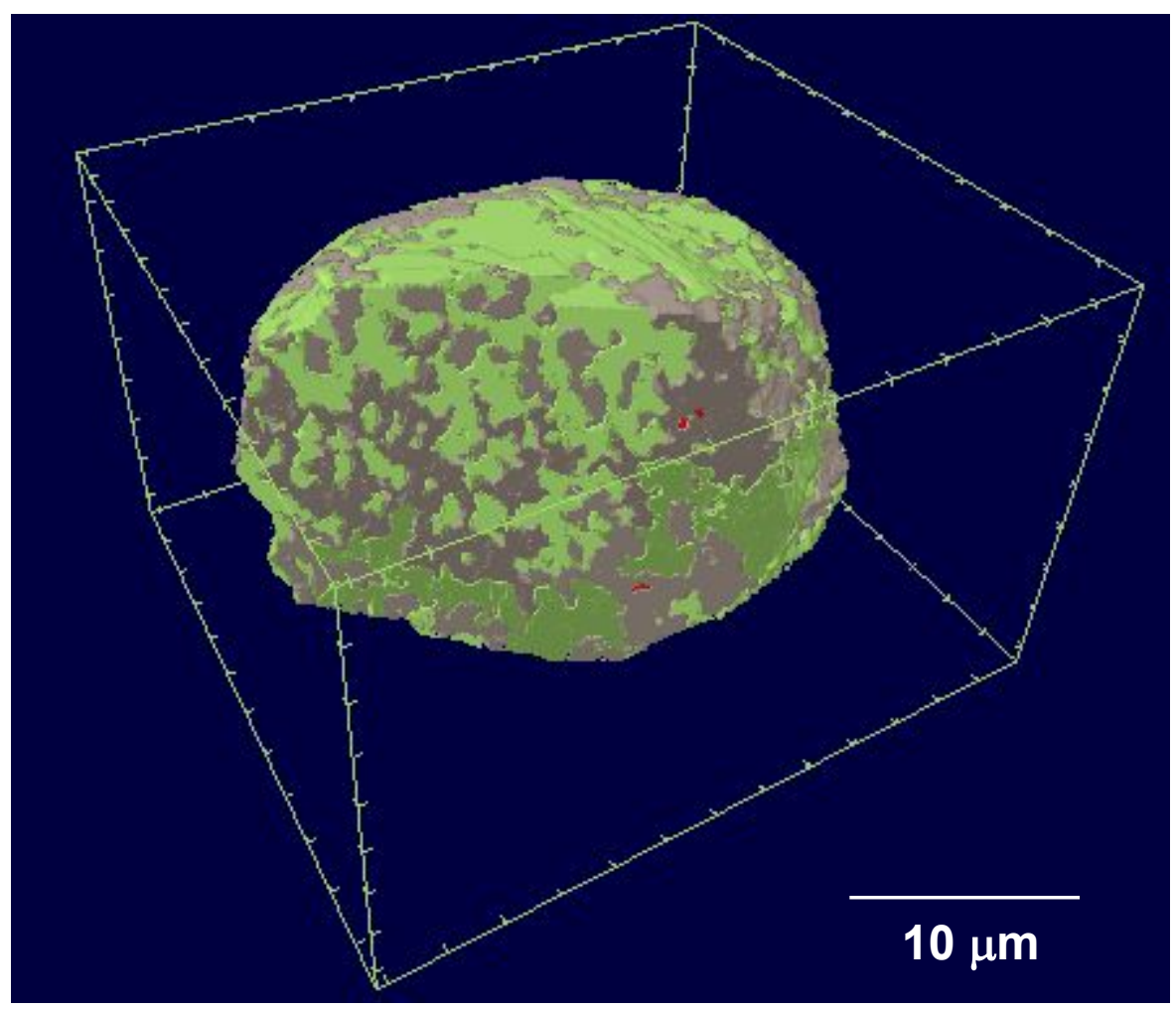

Figure S4. C-T Scan image of dust particle. Green area shows porous and cavity cites on dust particle. 
S3. Variation of saliva residue with dust size factor

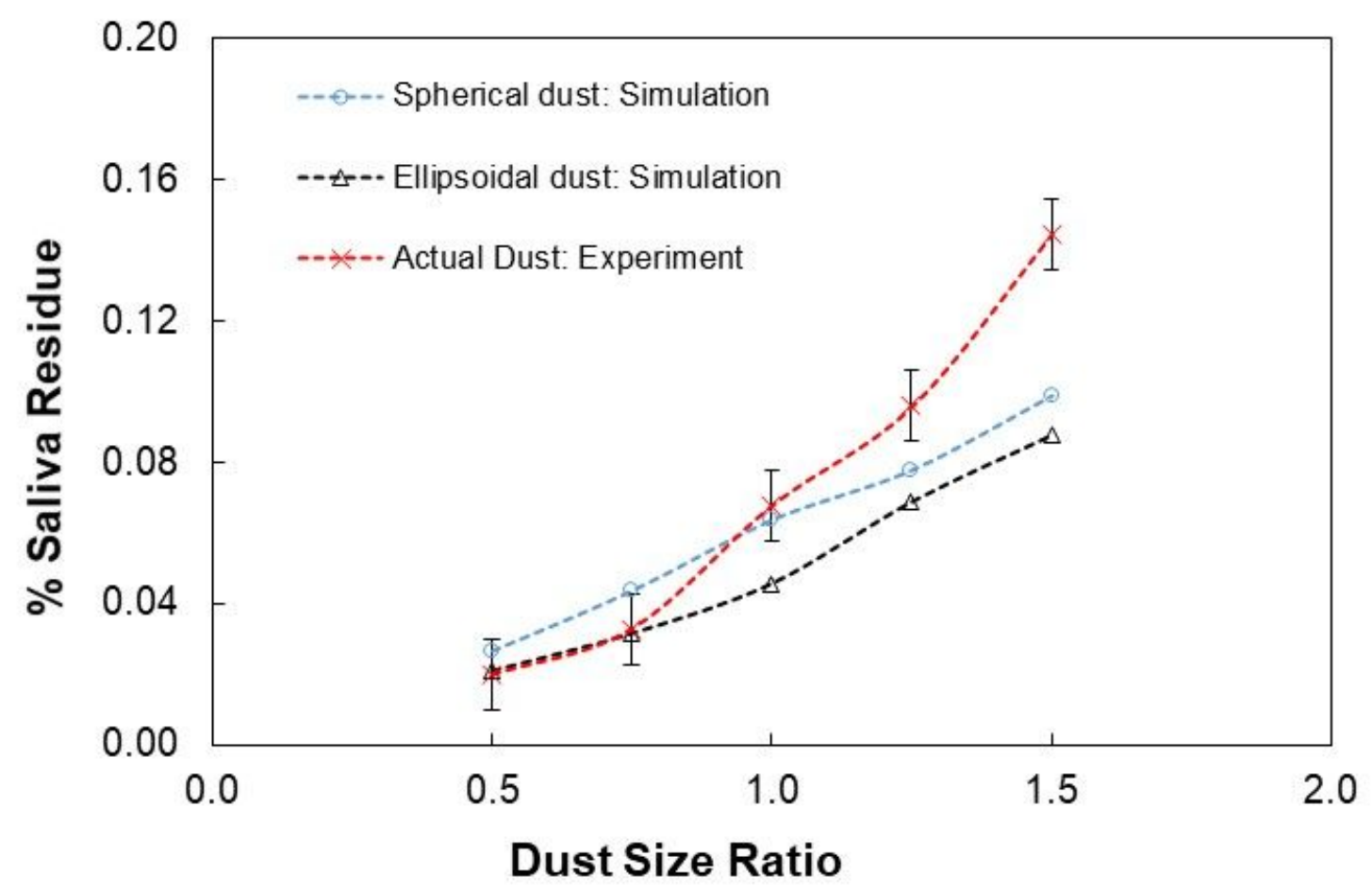

Figure S5. Variation of saliva residue with dust size factor obtained from simulations and experiment. 


\section{S4. References}

1. S Šikalo, Š., Wilhelm, H.-D., Roisman, I. V, Jakirlić, S., \& Tropea, C. Dynamic Contact Angle of Spreading Droplets: Experiments and Simulations. Phys. Fluids, 17(6), 2005.

2. Hu, J., Xiong, X., Xiao, H., and Wan, K. Effects of Contact Angle on the Dynamics of Water Droplet Impingement. COMSOL Conference, 2015.

3. COMSOL Inc. COMSOL Multiphysics. 2017. 